\title{
Depletive and repletive autoshaping schedules
}

\author{
GRAHAM C. L. DAVEY, BRIAN LEIGHFIELD, and GARY G. CLELAND \\ The City University, London, United Kingdom
}

\begin{abstract}
An experiment investigated the effects of depletive and repletive autoshaping schedules on signal-directed behavior in rats. Subjects that experienced a repletive schedule, in which the probability of the conditioned stimulus's being followed by response-independent food increased throughout the session, exhibited a gradual increase in signal-directed responding throughout a session, and contacted the conditioned stimulus significantly more often than did nonpaired control subjects. Rats that experienced a depleting schedule, in which the probability of the conditioned stimulus's being followed by food decreased throughout a session, showed a decreasing trend in signal-directed behavior during a session. Nonpaired control groups showed no within-session trends in conditioned stimulus contacts.
\end{abstract}

When a localizable conditioned stimulus (CS) is paired with an appetitive reinforcer or an unconditioned stimulus (UCS), animals frequently approach and contact the CS. This phenomenon has variously been called autoshaping (Brown \& Jenkins, 1968) and signal-directed or signal-centered behavior (Jenkins, Barrera, Ireland, \& Woodside, 1978). Although it is known that autoshaping obeys Pavlovian associative rules (see Hearst, 1979), much less is known about the performance mechanisms and biological significance of this behavior. Some writers have suggested that the tendency to approach appetitive CSs is a functionally distinct class of Pavlovian conditioned responses (CRs) that has general adaptive value and hence maximizes the inclusive fitness of a very wide variety of species (see Hollis, 1982, and Wasserman, 1980); others, however (e.g., Davey \& Cleland, in press; Timberlake, 1983) have suggested that autoshaped responding reflects the release of components from the organism's functionally organized feeding behavior system and hence that signal-directed behaviors are more likely to be characteristic of species-specific foraging behaviors. This latter approach stresses that the Pavlovian CS has a dual function: (1) as a signal that the frequency of food availability is relatively high, and (2) as a natural releaser for modules of the animal's feeding behavior system that include approach-and-investigate components (see Davey \& Cleland, in press). Hence, rats approach and contact a retractable lever CS paired with food because the CS signals a high probability of food delivery and this primes the lever (a small manipulable object) to release approach-and-investigate foraging responses. One of the implications of this type of account is that if autoshaping is behavior from an animal's food-foraging repertoire, then autoshaped

The authors' mailing address is: Department of Social Science and Humanities, The City University, London EC1V OHB, United Kingdom. responding should exhibit some of the more general characteristics of foraging behavior. In the wild, animals rarely encounter the constant contingency between signal and food that they experience in the Pavlovian conditioning experiment: Environmental stimuli signal either a decreasing density of food as the animal consumes prey in that area (a depleting "patch" of food) or, more rarely, an increasing density of food as the animal scares up prey during its foraging (a repletive "patch" of food). Furthermore, unlike in the prototypical Pavlovian conditioning experiment, food is usually available from other sources and is signaled by alternative cues. One of the more successful attempts to predict the strength of foraging in more natural conditions is optimal foraging theory (see Charnov, 1976, and Krebs, 1978). This theory suggests that the strength or rate of foraging in one set of stimulus conditions (a "patch") is a function of the probability of finding food under those conditions. To all intents and purposes, a "patch" can be conceived of as a Pavlovian CS that predicts a static (or changing) density of food. One of the implications of optimal foraging theory is that animals are continually monitoring the relative density of food in differing stimulus conditions and adjusting their foraging accordingly. If autoshaped responding represents species-specific foraging, then the strength of this behavior should also vary with the animal's moment-tomoment assessment of the predictive significance of the CS. The experiment described in this paper represents an attempt to construct depleting and repletive autoshaping schedules and to observe the effect of these schedules on the strength of signal-directed behavior in rats.

\section{METHOD}

Subjects
The subjects were 24 male hooded Lister rats maintained at
$80 \%$ of their ad-lib feeding weights during training. All were 
experimentally naive and were approximately 90 days old at the outset of the experiment.

\section{Apparatus}

Training was carried out in Campden Instruments rat conditioning chambers fitted with retractable levers. The dimensions of these chambers were outlined in Davey, Oakley, \& Cleland (1981). Situated in one wall of the chamber was a central foodtray recess $5.0 \mathrm{~cm}$ high and $4.0 \mathrm{~cm}$ wide. A Perspex flap covered this recess and recorded tray entries via a microswitch that operated when the door was pushed. Reinforcement was provided in the form of a single $45-\mathrm{mg}$ food pellet. All reinforcer deliveries were accompanied by a short flash of the food-tray light. Situated $3.0 \mathrm{~cm}$ to the left of the food tray was a retractable lever that could be extended $2.2 \mathrm{~cm}$ into the chamber. When retracted, the lever was flush with the wall of the chamber, and when extended into the chamber, lever contacts could be recorded by means of a drinkometer circuit connected to the lever. The lever took approximately $.5 \mathrm{sec}$ to extend fully into the chamber. The trials were timed from the onset of insertion to the onset of retraction. Leverpresses were also recorded; a force of $0.78 \mathrm{~N}$ was required to operate the lever.

A small houselight situated on the ceiling of each chamber provided general illumination throughout each session. The chambers were in sound-attenuating boxes, the front doors of which were open to permit observation of the subjects through closed-circuit TV. A closed-circuit TV camera was positioned in front of each box throughout the entire experiment to relay TV pictures of the subjects to observers in an adjoining room. The experiment was controlled and data were collected by an Acorn Econet microcomputer using the ONLIBASIC language.

\section{Procedure}

Magazine training. For the first 5 days, all subjects received magazine training in which $45-\mathrm{mg}$ food pellets were delivered into the food tray on a variable-time (VT) 60 -sec schedule. Each session lasted for about $30 \mathrm{~min}$, and at the end of these five sessions all subjects were reliably taking the food pellet soon after it had been delivered.

After magazine training, the subjects were divided into four groups of six subjects each. These four groups were called depletive experimental, repletive experimental, depletive control, and repletive control. All subjects were given 18 sessions of training.

Depletive experimental group. During a session, the retractable lever (CS) was presented on 60 occasions (trials) at a mean intertrial interval of $60 \mathrm{sec}$ (minimum $=30 \mathrm{sec}$, maximum $=$ $90 \mathrm{sec}$ ). On each trial, the lever was inserted into the chamber for a period of $10 \mathrm{sec}$. On Trial 1 , the probability of the lever presentation being immediately followed by a food delivery was $p=1.0$. This probability then declined on each subsequent trial by a factor of $p=.02$, such that by Trial 50 the probability of food following the lever presentation was $p=0$. The probability of food following lever presentation remained at $p=0$ for the last 10 trials of a session. Food deliveries were also presented during the intertrial periods. Intertrial intervals were divided into 10 -sec periods, and the probability of a food delivery at the end of each of these $10-\mathrm{sec}$ periods was set at $\mathrm{p}=.2$ throughout the whole session. Thus, for this group, the rate of food delivery during intertrial periods remained at a relatively low constant level, but the probability of food at the end of each lever-insertion trial declined throughout the session.

Repletive experimental group. These subjects also had 60 trials per session, except that the probability of food following a lever insertion increased throughout the session. For the first 10 trials, the probability of food following a trial was fixed at $p=0$. From Trial 11 onward, the probability of food following a trial increased by a factor of $p=.02$ until, by the final trial of a session, $p=1.0$. Like the depletive group, this group also received food during intertrial periods, with $\mathrm{p}=.2$ at the end of each 10 -sec period of the intertrial interval.
Depletive control group. Like the depletive experimental group, this group received 60 lever presentations per session and an intertrial food delivery probability of $p=.2$ per $10-\mathrm{sec}$ period. However, lever insertions and the food deliveries that had been programmed on a depleting schedule to follow these insertions in the experimental group were here programmed on identical, but independent, VT 60 -sec schedules. Hence, this group acted as an upaired control group that received the same distribution of food throughout a session, but none of these deliveries were explicitly paired with the lever.

Repletive control group. This group acted as an unpaired control for the repletive experimental group in the same way as did the depletive controls for the depletive experimental group.

Observation procedures. On the final session of training, the lever-directed behavior of subjects during lever insertions was analyzed according to a number of preselected topographic categories. These categories were: (1) orienting-a rapid movement of the head toward the lever without contacting the lever; (2) sniffing-moving the nose around the lever while moving the vibrissae in a manner characteristic of sniffing an object; (3) mouthing-touching the lever with the mouth and making small nibbling movements; (4) licking-contacting the lever with the tongue; (5) biting-grasping the lever between the teeth; and (6) pawing-placing a paw on top of the lever or grasping the lever between the paws.

Each category was scored in terms of the percentage of trials with at least one instance of the behavior. One observer scored the responses that make up the reported data, but two observers had independently scored the responses on early sessions with $85 \%$ agreement between them, suggesting that the selected categories were reliable and objectively definable.

\section{RESULTS}

Figure 1 shows the rates of lever contacts, leverpresses, and tray entries for all four groups across the course of the final session of training. The repletive experimental group showed an increasing trend in lever contacting across the six 10-trial blocks [Page's L test for trend $L(K=6 ; N=6)=503, p<.01$ ] and an increasing trend in leverpressing across trial blocks $[\mathrm{L}(\mathrm{K}=6 ; \mathrm{N}=6)=$ $386, p<.01]$. The depletive experimental group showed a significant decreasing trend in lever contacting only across the last five of the six trial blocks $[\mathrm{L}(\mathrm{K}=5 ; \mathrm{N}=6)=$ $322, \mathrm{p}<.01]$ and, similarly, a decreasing trend in leverpressing across the last five trial blocks $[\mathrm{L}(\mathrm{K}=5$; $\mathrm{N}=6)=295, \mathrm{p}<.05]$. Neither the depletive control group nor the repletive control group showed any significant trend across trials in either lever contacting or leverpressing ( $p>.05)$. For the two repletive groups, lever contacts showed a significant trials $\mathrm{x}$ conditions interaction $[F(5,50)=4.435, \mathrm{p}<.05]$. Pairwise comparisons of the six trial blocks showed that in all six blocks the repletive experimental group exhibited a higher rate of lever contacting than did the respective control group (Tukey's $Q \gg>10.89, p<.01$ in all cases). There was, however, no significant trials $\mathrm{x}$ conditions interaction with lever contacts for the depletive groups $[F(5,50)=1.005, p>.05]$.

Figure 2 illustrates the probability of each of the five lever-contact topographies (except licking, which was not observed) over the six 10-trial blocks of Session 18 . For the repletive experimental group, there was an 


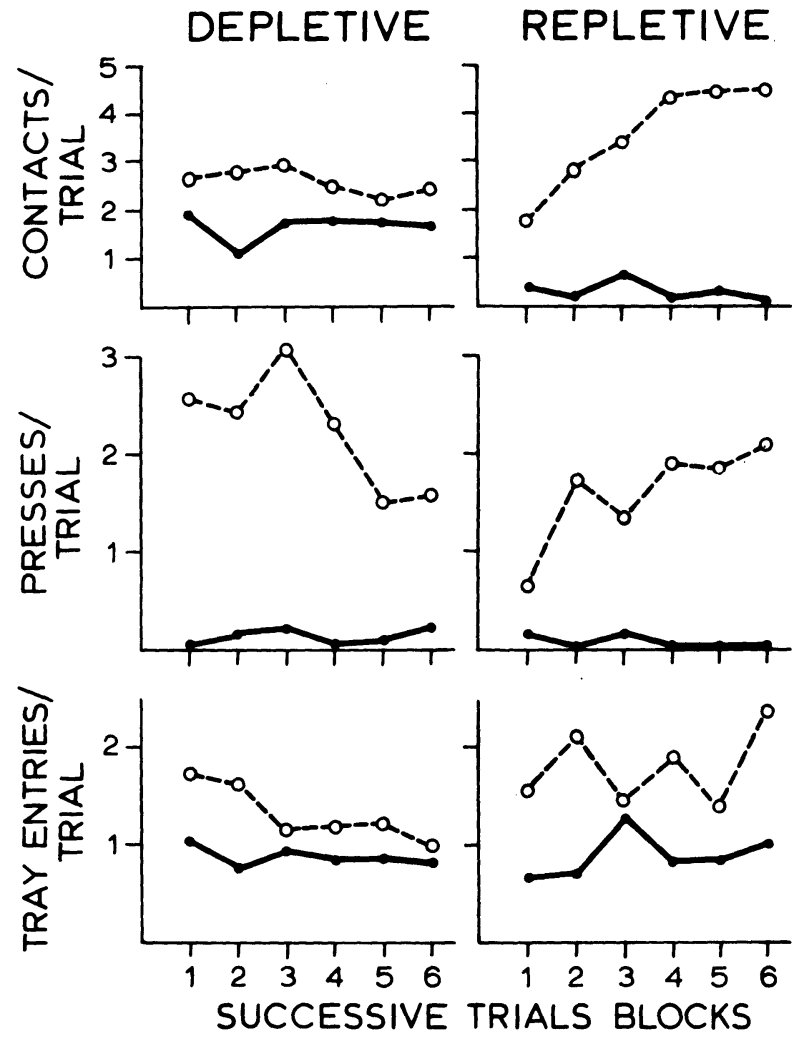

Figure 1. Rate of lever contacting, leverpressing, and foodtray entry during lever presentation for all four groups of subjects. Experimental groups $=$ open circles; control groups $=$ filled circles. The data are taken from the last session of training and are grouped into successive 10-trial blocks.

increasing trend across the session in all topographies except biting $(p<.05)$. For the depletive experimental group, there was a decreasing trend in all topographies across the session $(p<.05)$. There were no significant trends in the probability of topographies for either of the control groups ( $p>.05$ in all cases).

\section{DISCUSSION}

The results of this experiment suggest that (1) during the course of an autoshaping session, the strength of signal-directed behavior is a function of the probability with which the CS predicts food delivery; as this probability changes from trial to trial, so does the probability of a signal-directed action; (2) these changes in the strength of autoshaped responding were not determined solely by the changes in the frequency of food delivery that occurred during the session, since the nonpaired control groups-which received a similar patterning of food deliveries to the experimental groups-did not exhibit any significant within-session trends in autoshaped responding.

Contemporary theories of response strength, such as contingency theory (e.g., Rescorla, 1967) would predict that response strength in the two experimental groups should come to approximate that in their respective control groups when probability of food during lever presentation comes to equal the probability of food during intertrial intervals [i.e., when $\operatorname{Pr}($ UCS $/ C S)=\operatorname{Pr}(\mathrm{UCS} / \overline{\mathrm{CS}})]$. For the depletive group, this should be from Trial 40 onward, and for the repletive group, it should be prior to Trial 20. During these periods, the probability of food following a lever presentation would be lower than the probability $(p=.2)$ of food at the end of each 10 -sec intertrial interval. Similarly, from the viewpoint of optimal foraging theory, if the CS is considered as a temporal "patch" of food as seen against the changing availability of food in the habitat as a whole (a session), then the marginal value theory (Charnov, 1976) would predict that foraging (signal-directed behavior) should cease when the probability of food during the CS equals the probability of food in the habitat as a whole. On this basis, differential autoshaped responding should cease from around Trial 35 for the depletive group and commence from around Trial 25 for the repletive group. Neither the predictions from the contingency theory nor those from the marginal value theory were upheld in the present study. The reasons for this are unclear: The two repletive groups showed a significant difference in lever-contact rate from the first 10-trial block onward, and the depletive groups failed to exhibit a significant trials $\mathrm{x}$ conditions interaction.

Nevertheless, although performance models of autoshaping are focusing increasingly on the similarity of autoshaped behaviors and naturally occurring species-specific foraging activities, it seems heuristically fruitful to explore further how notions from foraging theory might cast light on the mechanisms underlying signal-directed responding and, indeed, how more naturally occurring contingencies (such as depletive and repletive schedules) affect response strength.

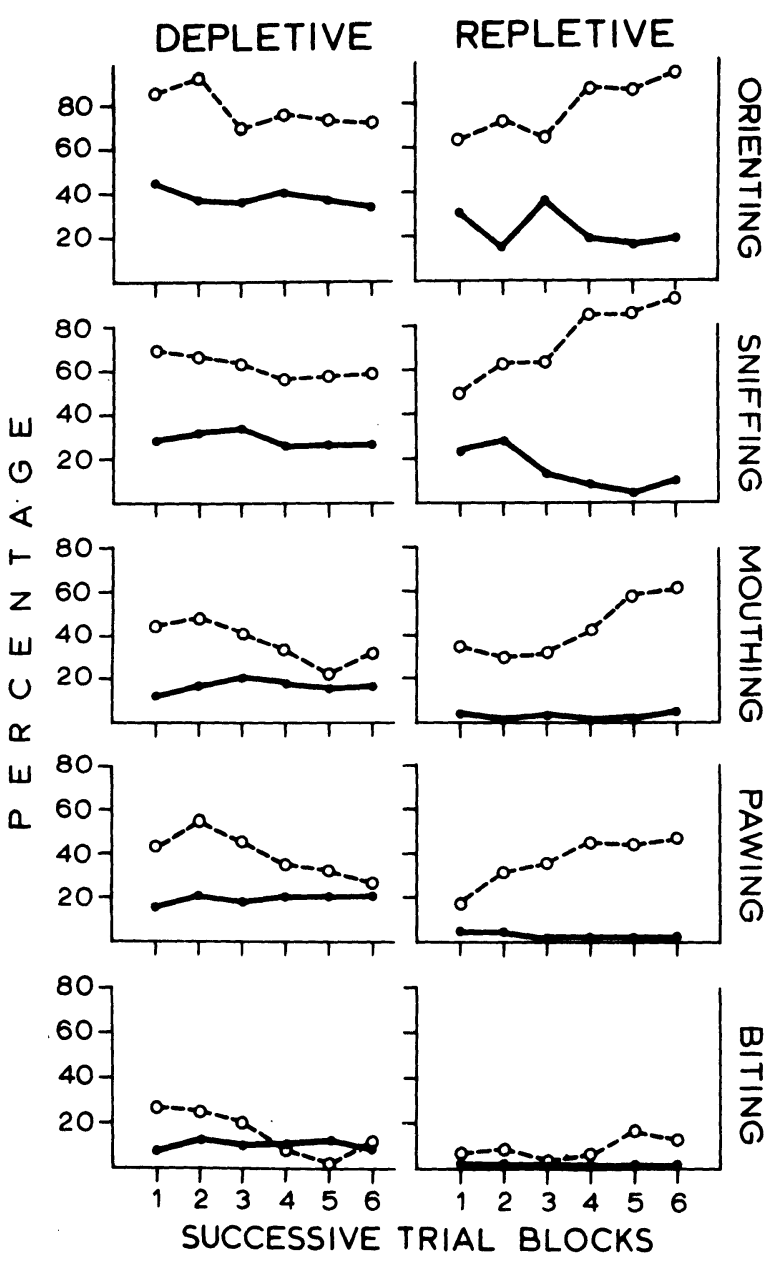

Figure 2. Mean percentage of trials with at least one occurrence of a particular response topography. Experimental groups = open circles; control groups = filled circles. The data are taken from the last session of training and are grouped into successive 10-trial blocks. 


\section{REFERENCES}

Brown, P. L., \& Jenkins, H. M. (1968). Auto-shaping of the pigeon's key-peck. Journal of the Experimental Analysis of Behavior, 11, 1-8.

Charnov, E. L. (1976). Optimal foraging: The marginal value theorem. Theoretical Population Biology, 9, 129-136.

Davey, G. C. L., \& Cleland, G. G. (in press). Food anticipation and lever-directed activities in rats. Learning and Motivation.

Davey, G. C. L., Oakley, D. A., \& Cleland, G. G. (1981). Autoshaping in the rat: Effects of omission on the form of the response. Journal of the Experimental Analysis of Behavior, 36, 75-91.

Hearst, E. (1979). Classical conditioning as the formation of interstimulus associations: Stimulus substitution, parasitic reinforcement and autoshaping. In A. Dickinson \& R. A. Boakes (Eds.), Mechanisms of learning and motivation. Hillsdale, NJ: Erlbaum.

Hollis, K. L. (1982). Pavlovian conditioning of signal-centered action patterns and autonomic behavior: A biological analysis of function. In J. S. Rosenblatt, R. A. Hinde, C. Beer, \& M. C. Busnel (Eds.), Advances in the study of behavior (Vol. 12). New York: Academic Press.

Jenkins, H. M., Barrera, F. J., Ireland, C., \& Woodside, B. (1978). Signal-centered action patterns of dogs in appetitive classical conditioning. Learning and Motivation, 9, 272-296.

KREBS, J. R. (1978). Optimal foraging: Decision rules for predators. In J. R. Krebs \& N. B. Davies (Eds.), Behavioral ecology: An evolutionary approach. Oxford: Blackwell.

Rescorla, R. A. (1967). Pavlovian conditioning and its proper control procedure. Psychological Review, 74, 71-80.

Timberlake, W. (1983). The functional organization of appetitive behavior: Behavior systems and learning. In M. D. Zeiler \& P. Harzem (Eds.), Advances in analysis of behavior (Vol. 3): Biological factors in learning. Chichester, England: Wiley.

WASSERman, E. A. (1980). Response evocation in autoshaping: Contributions of cognitive and comparative-evolutionary analyses to an understanding of directed action. In C. M. Locurto, H. S. Terrace, \& J. Gibbon (Eds.), Autoshaping and conditioning theory. New York: Academic Press.

(Manuscript received for publication December 13, 1983.)

\section{ERRATUM}

Calef, R. S., Metz, R. A., Atkinson, T. L., Pellerzi, R. C., Taylor, K. S., \& Geller, E. S. (1984). "Acquisition of running in the straight alley following experience with responseindependent food" [Bulletin of the Psychonomic Society, 22(1), 67-69]-On page 69, part of the paragraph in progress at the beginning of column 1 was misprinted-after "Hence, Group NC may have learned to approach in the presence of frustration-eliciting stimuli." We regret this error and extend our apologies to the authors and readers. The complete text for the partial paragraph in question (that is, the first 17 lines of page 69) should be:

pate food in the goal cup of the operant chamber. If an approach response in the operant chamber was followed by no food, frustration may have occurred and become conditioned to the chamber cues. Conditioned frustration (rf-sf) elicited by the chamber cues may occasionally have been followed by reward in the goal cup. Hence, Group NC may have learned to approach in the presence of frustration-eliciting stimuli. According to Amsel's (1972) persistence theory, learning to approach in the presence of aversive stimuli (rf-sf) should cause subjects to persist in the presence of any disrupting stimuli, whether those stimuli are novel stimuli elicited by an apparatus during the acquisition of a new response or rf-sf during extinction. Hence, rats receiving response-independent food should have acquired the running response faster in the present study than did the control rats because of the generalization of learning to persist in the presence of a disruptive stimulus, and should have shown more resistance to extinction in the Calef et al. study. 